Received: 2021/10/19, Revised: 2021/12/05, Accepted: 2021/12/23, Published: 2021/12/31 @2021 Bok Sil Hong et al.; Licence Physical Activity and Nutrition. This is an open access article distributed under the terms of the creative commons attribution license (https:/l creativecommons.org/licenses/by-nc/2.0/), which permits unrestricted use, distribution, and reproduction in any medium, provided the orginal work is properly cited.

${ }^{*}$ Corresponding author : Jisu Kim and Kang Pa Lee

Jisu Kim

Department of Medical Science, School of Medicine/Physical Activity \& Performance Institute, Konkuk University, 120 Neungdong-ro, Gwangin-gu, Seoul 143-701, Republic of Korea

Tel: +82-10-2039-4225

Fax: +82-0504-485-4225

E-mail: kimpro@konkuk.ac.kr

Kang Pa Lee

Research \& Development Center, UMUST R\&D corporation, 84, Madeul-ro 13-gil, Dobong-gu, Seoul (01411) Republic of Korea

Tel: +82-2-998-5552

E-mail: umustrnd@naver.com

(2021 The Korean Society for Exercise Nutrition

[Purpose] Exercise can prevent conditions such as atrophy and degenerative brain diseases. However, owing to individual differences in athletic ability, exercise supplements can be used to improve a person's exercise capacity. Schisandra chinensis (SC) is a natural product with various physiologically active effects. In this study, we analyzed SC using a pharmacological network and determined whether it could be used as an exercise supplement

[Methods] The active compounds of SC and target genes were identified using the Traditional Chinese Medicine Database and Analysis Platform (TCMSP). The active compound and target genes were selected based on pharmacokinetic (PK) conditions (oral bioavailability $(\mathrm{OB}) \geq 30 \%$, Caco-2 permeability (Caco-2) $\geq$ -0.4 , and drug-likeness $(\mathrm{DL}) \geq 0.18$ ). Gene ontology $(\mathrm{GO})$ was analyzed using the Cytoscape software.

[Results] Eight active compounds were identified according to the PK conditions. Twenty-one target genes were identified after excluding duplicates in the eight active compounds. The top 10 GOs were analyzed using GO-biological process analysis. GO was subsequently divided into three representative categories: postsynaptic neurotransmitter receptor activity $(53.85 \%)$, an intracellular steroid hormone receptor signaling pathway $(36.46 \%)$ and endopeptidase activity $(10 \%)$. SC is related to immune function.

[Conclusion] According to the $\mathrm{GO}$ analysis, SC plays a role in immunity and inflammation, promotes liver metabolism, improves fatigue, and regulates the function of steroid receptors. Therefore, we suggest SC as an exercise supplement with nutritional and anti-fatigue benefits.

[Keywords] Schizandra chinensis, neurotransmitter, immune system, liver metabolism, anti-fatigue, exercise supplement

\section{Systematic analysis of the pharmacological function of Schisandra as a potential exercise supplement}

\author{
Bok Sil Hong ${ }^{1,2}$ / Suji Baek ${ }^{3}$ Myoung-Ryu Kim¹ / Sun Mi Park ${ }^{4}$ / \\ Bom Sahn Kim ${ }^{4}$ Jisu Kim ${ }^{5,6^{*}} /$ Kang Pa Lee ${ }^{3,4^{*}}$ \\ 1. Department of Nursing, Cheju Halla University, Jeju, Republic of Korea \\ 2. Life Science Research Center, Cheju Halla University, Jeju, Republic of Korea \\ 3. Research and Development Center, UMUST R\&D Corporation, Seoul, Republic of Korea \\ 4. Department of Nuclear Medicine, Ewha Womans University College of Medicine, Seoul, Republic of Korea \\ 5. Physical Activity \& Performance Institute, Konkuk University, Seoul, Republic of Korea \\ 6. Department of Sports Medicine and Science in Graduated School, Konkuk University, Seoul, Republic of \\ Korea
}

\section{INTRODUCTION}

The increasing incidence of chronic diseases in elderly adults has gained attention owing to an increase in the elderly population ${ }^{1}$. Older adults with chronic diseases such as Alzheimer's, Parkinson's, and heart diseases require considerable public health resources, and disease complications can cause substantial losses to the social environment ${ }^{2}$. To prevent degenerative neurological diseases in elderly adults and improve their health, there is an urgent need to determine practical measures that can maintain a healthy quality of life. Increasing scientific evidence has demonstrated an association between exercise and degenerative diseases in elderly adults ${ }^{3}$. Therefore, exercise or physical activity is the safest and most effective anti-aging treatment and can prevent cardiovascular, musculoskeletal, and age-related brain diseases.

Exercise can suppress various diseases; however, continuous exercise may be difficult owing to differences in an individual's capacity to continue exercising ${ }^{4}$. Interest in the development of natural products has recently increased, and a wide variety of research has been conducted, ranging from the investigation of raw and functional materials from animals and plants to the development of new substances that have positive effects on physiological activities ${ }^{5,6}$. Most studies on the development of these natural substances have focused on their function as supplements that can provide beneficial pharmacological effects ${ }^{7}$. Furthermore, these substances need to be harmless to the human body, utilizing easily obtained raw materials with a simple production regime. Verifying the magnitude of enhancements to exercise performance rates and identifying the action mechanisms of these supplements can improve the physical activity rates of the elderly in addition to the performance of athletes ${ }^{8}$. Therefore, it is necessary to develop sports drinks or supplements to improve participation in physical activity.

$\mathrm{SC}$, also known as Omjia in South Korea, is a woody plant of the Magnolia family that is mainly distributed in the central and northern regions of South Korea9 ${ }^{9}$. It has a unique aroma, strong sour taste, and high 
tannin content. In addition, SC has demonstrated anti-inflammatory and antioxidant properties through its pharmacological functions, resulting in hepatoprotective effects ${ }^{10,11}$. As SC is expected to prevent brain disease and improve athletic performance ${ }^{12}$, it may be a candidate substance for exercise supplements. However, there are insufficient data on the target protein activities of SC in the human body as limited molecular-level network analyses are available. Therefore, based on the genes regulated by SC, we investigated the most significant mechanisms for addressing brain disease and improving exercise activity through a systemic analysis of its pharmacological functions in exercise supplements.

\section{METHODS}

Identification and construction of a database of the active ingredients in SC

The Traditional Chinese Medicine Systems Pharmacology Database and Analysis Platform (TCMSP; http://ibts. hkbu.edu.hk/LSP/tcmsp.php) was used to identify the active ingredients in $\mathrm{SC}^{13}$. As recommended by TCMSP, the bioactive substances were analyzed based on their pharmacokinetic properties (absorption, distribution, metabolism, and excretion (ADME) of a drug), including oral bioavailability (OB), Caco-2 permeability (Caco-2) for intestinal epithelial permeability, and drug-likeness (DL). For further analysis, the active compounds with $\mathrm{OB} \geq 30 \%$, Caco- $2 \geq-0.4$, and $\mathrm{DL} \geq 0.18$, were selected as candidate compounds.

\section{Construction of an "active ingredients-potential targets" SC network}

The active components of SC perform related biological functions through specific targets. After obtaining the potential targets of the core active ingredients in Omjia directly from the TCMSP database, the protein names of all target genes were converted into corresponding gene symbols using the UniProt database. Gene ontology (GO) analysis was then performed to identify biological processes related to the collected genes using the DAVID 6.8 Gene Functional Classification Tool (https://david.ncifcrf.gov/). The $p$-values of the enrichment results were corrected using the Benjamini-Hochberg method, and significance of enriched values were set at $p<0.01$.

\section{Target network analysis of SC bioactive substances}

To analyze and understand the mechanisms of SC based on active ingredients and target networks, the compoundtarget networks were constructed using the Cytoscape visualization software 3.7 .2 (https://cytoscape.org/). The selected candidate ingredients and targets were input into the software, and the network was analyzed. The relationships between various active SC ingredients and target genes as well as the biological metabolic processes related to exercise metabolism were selected, and a network (process-target network, PT network) was subsequently constructed.

\section{RESULTS}

\section{Identification of active compounds}

First, we constructed a compound-target network at the molecular level to determine the regulatory factors of the active ingredients of SC using the TCMSP database. Table 1 shows these eight bioactive SC compounds, including Longikaurin A, Deoxyharringtonine, Angeloylgominsin O, Gomisin-A, -G, and -R. Eight out of a total of 130 active compounds in SC met the criteria of $\mathrm{OB} \geq 30 \%$, caco- $2 \geq$ -0.4 , and $\mathrm{DL} \geq 0.18$.

Table 1. Active compounds of Schizandra chinensis (SC).

\begin{tabular}{|ccccc}
\hline & Molecule name & OB (\%) & Caco-2 & DL \\
\hline 1 & Longikaurin A & 47.72 & 0.08 & 0.53 \\
\hline 2 & Deoxyharringtonine & 39.27 & 0.19 & 0.81 \\
\hline 3 & Angeloylgomisin & 31.97 & 0.83 & 0.85 \\
\hline 4 & Schizandrer B & 30.71 & 0.66 & 0.83 \\
\hline 5 & Gomisin-A & 30.69 & 0.63 & 0.78 \\
\hline 6 & Gomisin G & 32.68 & 0.73 & 0.83 \\
\hline 7 & Gomisin R & 34.84 & 0.6 & 0.86 \\
\hline 8 & Wuweizisu C & 46.27 & 1.08 & 0.84 \\
\hline
\end{tabular}

* OB, oral bioavailability; Caco-2, Caco-2 permeability; DL, drug-likeness.

\section{Potential target genes from active compounds and SC}

We also analyzed genes related to the active molecules of SC using TCMSP. A total of 30 genes were associated with the eight active compounds, and 21 genes were ultimately identified, excluding duplicates (Table 2). As shown in Figure 1, we visualized the active molecule-target network using the Cytoscape software. The four active compounds, namely, Angelolygimisin O, Shizander B, Gomisin G, and Gomisin R, commonly contained DNA topoisomerase II- $\alpha$ (TOP2A) as a target gene. The three active compounds, Deoxyharringtonline, Angeloylgomisin O, and Gomisin R, commonly contained androgen receptor (AR) as a target gene.

Table 2. Potential target genes for the active compounds of Schizandra chinensis (SC).

\begin{tabular}{|c|c|c|}
\hline & Molecule name & Gene symbol \\
\hline 1 & Longikaurin A & $\begin{array}{c}\text { CHRM1, GABRA2, GABRA3, } \\
\text { CHRM2, PRSS1, GABRA6 }\end{array}$ \\
\hline 2 & Deoxyharringtonine & AR, NR3C2 \\
\hline 3 & Angeloylgomisin & AR, F10, TOP2A \\
\hline 4 & Schizandrer B & PTGS2, TOP2A \\
\hline 5 & Gomisin-A & F2, NOS3, ACHE \\
\hline 6 & Gomisin G & TOP2A \\
\hline 7 & Gomisin R & $\begin{array}{l}\text { ESR1, AR, F10, PTGS2, TOP2A, } \\
\text { PRSS1, PIM1, NCOA2, KCNMA1 }\end{array}$ \\
\hline 8 & Wuweizisu C & ACHE, GABRA1, DPP4, CYP450 \\
\hline
\end{tabular}




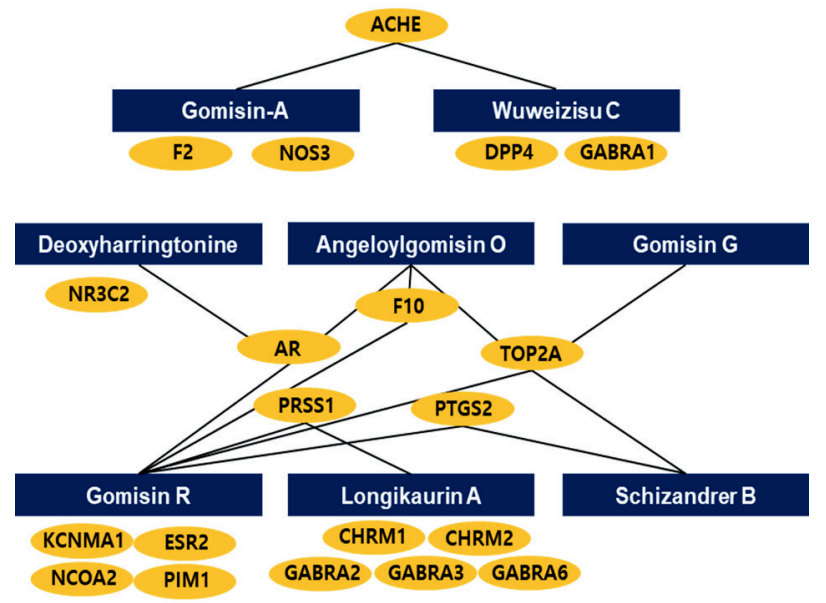

Figure 1. Gene network targeted by representative compounds of Schisandra chinensis (SC). The blue squares represent the eight active compounds of SC. The orange ellipses represent the target genes of the eight active compounds. The black lines link the genes corresponding to the active compounds.
G0 analysis of the gene network as targeted by representative compounds

To analyze the function of the $\mathrm{SC}$ active substances, the ontology of the 21 selected genes was analyzed using the GO biological processes function from the Cytoscape software. As shown in Figure 2, the 21 genes were associated with the top 13 GOs: endopeptidase activity, the intracellular steroid hormone receptor signaling pathway, negative regulation of apoptotic process, regulation of DNA-binding transcription factor activity, transcription initiation from the RNA polymerase II promoter, nuclear receptor activity, inhibitory extracellular lined-gated ion channel activity, GABA-gated chloride ion channel activity, chemical synaptic transmission, postsynaptic neurotransmitter receptor activity, chloride transmembrane transport, synaptic transmission cholinergic, and GABAergic synaptic transmission (Figure $2 \mathrm{~A}$ ). The most relevant GO was postsynaptic neurotransmitter receptor activity (Figure 2B). The genes associated with each GO are listed in Table 3 and Figure 3.

A

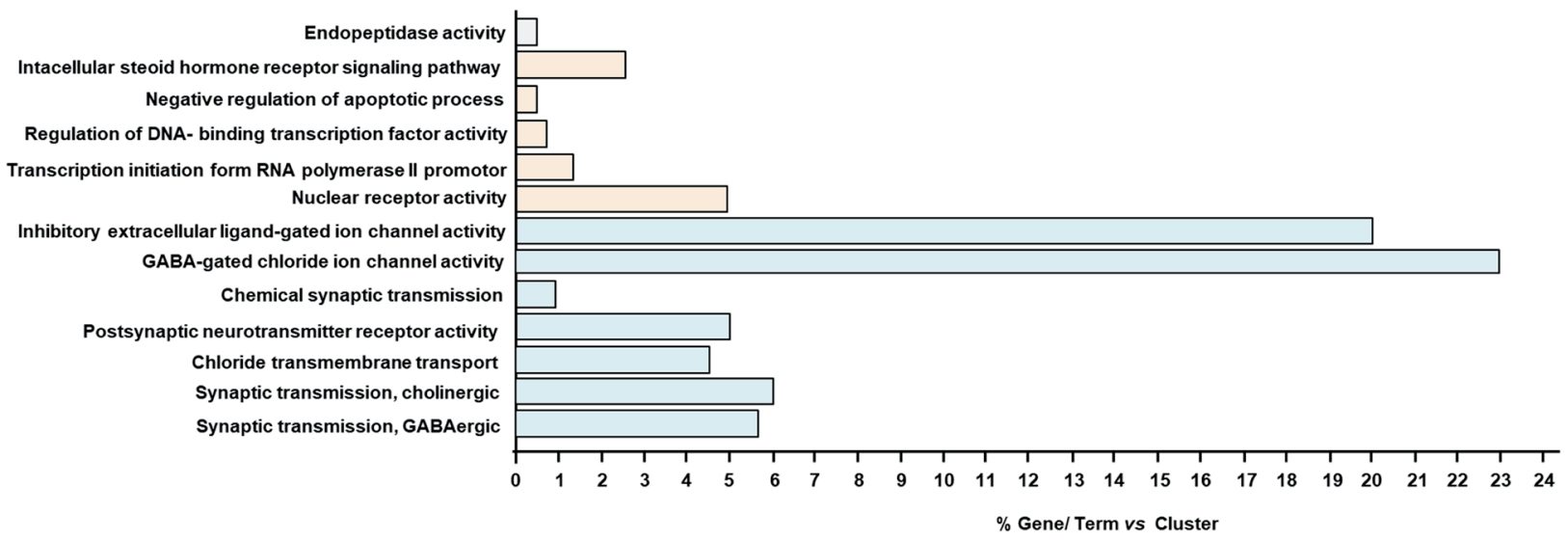

B

Figure 2. Gene ontology (GO) analysis of differentially expressed genes through the eight active compounds of Schisandra chinensis (SC). (A) The bar graph represents the highest expressions of GO using the Cytoscape software. (B) The pie graph represents the grouping of GOs in Panel A of the Cytoscape software. 
Table 3. Top 10 enriched biological processes from the gene ontology (GO) analysis.

\begin{tabular}{|c|c|c|c|c|}
\hline Category & Term & Molecule name & $P$-value & Gene \\
\hline GOTERM_BP & GO:0099528 & G protein-coupled neurotransmitter receptor activity & $2.0 \mathrm{E}-11$ & $\begin{array}{l}\text { ADRB1, CHRM1, CHRM2, } \\
\text { CHRM3, CHRM4, CHRM5 }\end{array}$ \\
\hline GOTERM_BP & GO:1904999 & positive regulation of leukocyte adhesion to arterial endothelial cell & $2.5 \mathrm{E}-6$ & ALOX5, TNF \\
\hline GOTERM_BP & GO:0004935 & adrenergic receptor activity & $2.0 \mathrm{E}-11$ & $\begin{array}{l}\text { ADRA1A, ADRA1B, ADRA1D, } \\
\text { ADRA2A, ADRB1, ADRB2 }\end{array}$ \\
\hline GOTERM_BP & GO:0001993 & $\begin{array}{l}\text { regulation of systemic arterial blood pressure by } \\
\text { norepinephrine-epinephrine }\end{array}$ & $2.0 \mathrm{E}-11$ & $\begin{array}{l}\text { ADRA1A, ADRA1B, ADRA1D, } \\
\text { ADRB1, ADRB2 }\end{array}$ \\
\hline GOTERM_BP & GO:0001996 & positive regulation of heart rate by epinephrine-norepinephrine & $2.0 \mathrm{E}-11$ & $\begin{array}{l}\text { ADRA1A, ADRA1B, } \\
\text { ADRA1D, ADRB1 }\end{array}$ \\
\hline GOTERM_BP & GO:0002025 & $\begin{array}{l}\text { norepinephrine-epinephrine-mediated vasodilation involved in } \\
\text { regulation of systemic arterial blood pressure }\end{array}$ & $2.0 \mathrm{E}-11$ & ADRB1, ADRB2 \\
\hline GOTERM_BP & GO:0004936 & alpha-adrenergic receptor activity & $2.0 \mathrm{E}-11$ & $\begin{array}{l}\text { ADRA1A, ADRA1B, } \\
\text { ADRA1D, ADRA2A }\end{array}$ \\
\hline GOTERM_BP & GO:0004939 & beta-adrenergic receptor activity & $2.0 \mathrm{E}-11$ & ADRB1, ADRB2 \\
\hline GOTERM_BP & GO:0001994 & $\begin{array}{l}\text { norepinephrine-epinephrine vasoconstriction involved in } \\
\text { regulation of systemic arterial blood pressure }\end{array}$ & $2.0 \mathrm{E}-11$ & ADRA1A, ADRA1D \\
\hline GOTERM_BP & GO:0001985 & $\begin{array}{l}\text { negative regulation of heart rate involved in baroreceptor } \\
\text { response to increased systemic arterial blood pressure }\end{array}$ & $2.0 \mathrm{E}-11$ & ADRA1A, ADRA1D \\
\hline
\end{tabular}

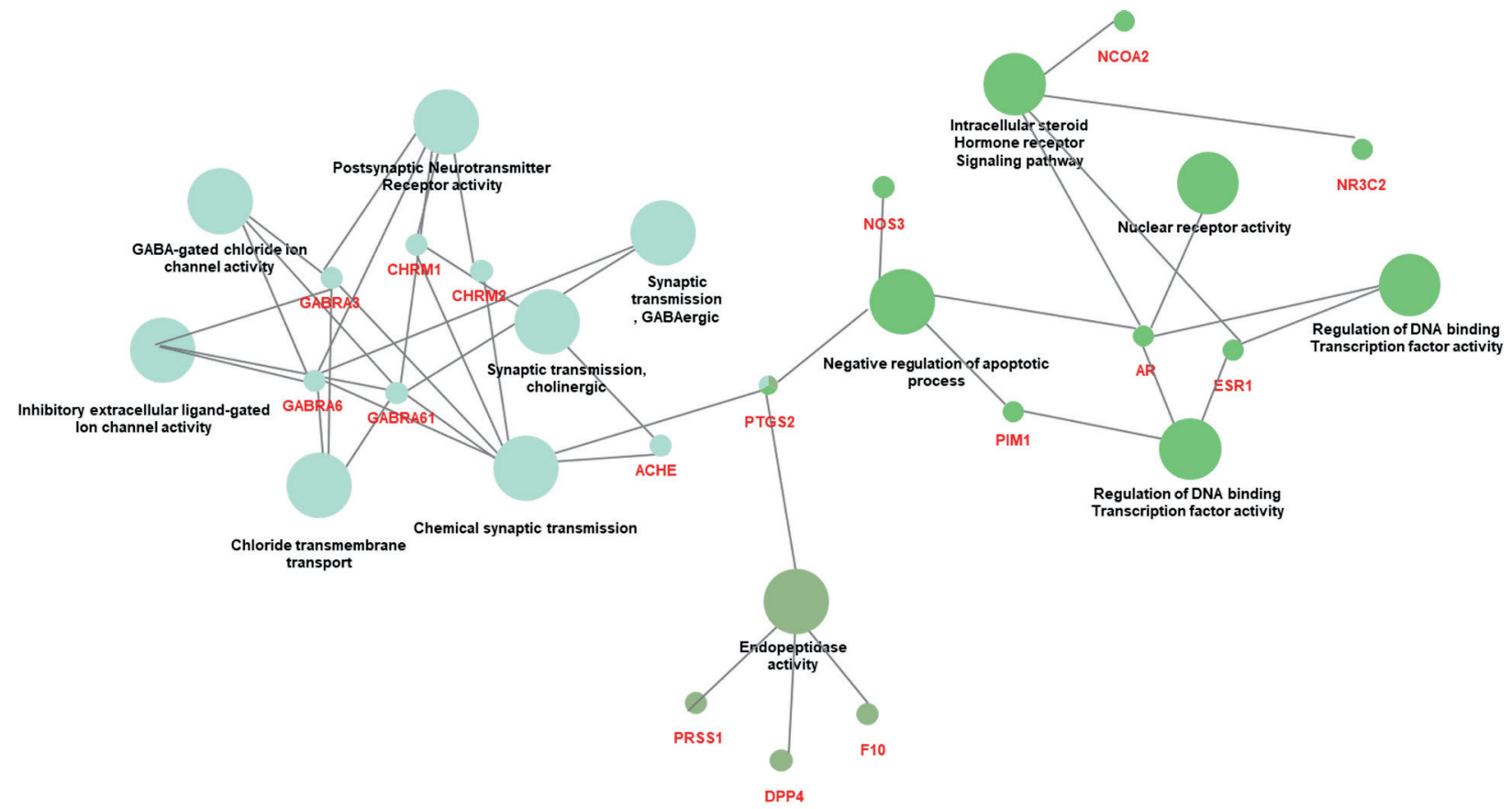

Figure 3. Biological processes of gene ontology (GO) via compound-target analysis of Schisandra chinensis (SC). The large circles indicate the categories as grouped by GO. Genes associated with biological networks are indicated in red letters.

\section{DISCUSSION}

The purpose of this study was to attempt to predict whether SC could function as a sports supplement to improve athletic performance. Therefore, we screened physiologically active substances of SC from the TCMSP database that can improve exercise capacity after a single intake, investigated target proteins related to its active ingredients, and performed a protein network analysis targeted by SC based on the results of the systematic analysis of their pharmacological functions. These results imply that SC active compounds such as Longikaurin A, Deoxyharringtonine, Angeloylgominsin O, Gomisin-A, -G, and, -R may control inflammation, promote liver metabolism, improve fatigue, and regulate the functions of steroid receptors.

According to the Korean traditional medical book, Dongui Bogam, SC is considered a wonder drug that cleans the blood by improving blood circulation and acts as a natural nourishment tonic with medical efficacy ${ }^{14}$. Several studies have suggested that SC exhibits anti-cancer, antioxidant, anti-inflammatory, and anti-fatigue benefits ${ }^{15,16}$. In a recent randomized, double-blind, placebo-controlled trial, quadri- 
ceps muscle strength (QMS) values significantly increased following SC administration, and lactate levels significantly decreased in healthy post-menopausal middle-aged women who consumed $1000 \mathrm{mg}$ of SC extract compared to the placebo group ${ }^{17}$. These effects may have resulted from increased ATPase activity and improved mitochondrial function following SC extract supplementation ${ }^{17}$. However, there are insufficient data on molecular level network analyses in the field of exercise metabolism and exercise nutrition. Interestingly, when identifying proteins related to the physiologically active substances of SC in our study, we observed that steroid receptor functions could be improved (Figure 2). Steroids are organic compounds that are used as signal transmitters in the body and produce androgen hormones to promote bone and muscle formation and to increase strength and energy ${ }^{18}$. Muscle strength and metabolism are highly important for maintaining health, especially for athletes because they are directly related to exercise abilities ${ }^{19,20}$. We suggest that the nourishing and tonic effects of SC can relieve the physical fatigue transmitted to the brain after a single uptake.

Inflammatory responses are important for the development of substances that address exercise impairment and various diseases ${ }^{21}$. Excessive exercise or muscle damage can lead to the accumulation of inflammatory factors in the body, thereby harming human health ${ }^{22}$. Acute and chronic inflammation are critical factors in disease development ${ }^{23}$. For example, cycloxygenase-2 (COX-2) is a major biomarker of inflammatory responses, and the expression level of COX rapidly increases when muscles are damaged by acute exercise or inflammation in the body ${ }^{24}$. SC extract leads to a significant decrease in LPS-induced macrophage inflammatory responses both in vitro and in vivo ${ }^{25}$. Our results indicated that SC interacts with the target protein, COX2. Therefore, after a single intake, the active ingredients of SC are expected to improve the capacity and duration of exercise by inhibiting inflammatory responses.

Systemic inflammatory reactions can decrease exercise capacity and increase exercise inactivity ${ }^{26}$. Furthermore, with an increase in obesity and overweight populations because of by overeating habits and sedentary lifestyles, the incidence of diabetes is increasing worldwide 27,28 . Metabolic diseases, such as type 2 diabetes mellitus (DM) and nonalcoholic fatty liver disease, are gradually threatening the health of the global population ${ }^{29,30}$. Several studies have suggested that exercise can mitigate metabolic diseases by regulating the molecular levels ${ }^{31,32}$. For example, dipeptidyl peptidase-4 (DPP-4), which is highly expressed in the liver, is a type of transmembrane peptidase that inhibits type 2 $\mathrm{DM}^{33}$. DPP-4 inhibition potentially improves hepatic steatosis and insulin resistance, similar to exercise $\mathrm{e}^{34,35}$. Our data confirmed that the active compound network in SC helps regulate DDP-4 expression. According to these combined analysis results, exercise after consuming SC can strengthen muscles through steroid signal transduction, promote metabolism, and control inflammation and diabetes.

In recent decades, numerous studies have been conducted, and various theories on the mechanisms of physical fatigue have been published. The generally accepted mechanistic insights are peripheral fatigue caused by energy depletion and central fatigue, which are the effects of neurotransmitters ${ }^{36}$. When physically exhausted, substances accumulated by sustained physical activity and high-intensity exercise fatigue stimulate neurotransmitters to signal the brain for rest ${ }^{37}$. Exercise avoidance is associated with signal transmission in response to fatigue ${ }^{38}$. Several studies have revealed the nourishing and tonic effects of $\mathrm{SC}^{39,40}$. The optimal intake of SC varies. In previous animal experiments, with a positive effect on skeletal muscle and biomarkers seen, a dose of $500 \mathrm{mg} / \mathrm{kg}$ ( $2430 \mathrm{mg} /$ day when converted to $60 \mathrm{~kg}$ human) of SC extract supplementation was recommended $^{41}$. However, recently published studies seem to view $1 \mathrm{~g}$ /day as the optimal dose. Cho et al. reported that SCs supplementation (1 g/day) can enhance skeletal muscle strength but not mass in older adults who perform low-intensity exercise $\mathrm{e}^{42}$. In addition, the results of Jang et al. have shown that supplementation with SC (1 g/day) may help to improve QMS and decrease lactate levels in adult women ${ }^{17}$. In a recent study, mice that ingested glycoprotein from SC demonstrated an extended swimming time owing to increased lactate dehydrogenase (LDH), superoxide dismutase (SOD), and glutathione peroxidase (GSH-Px) activities, greater liver glycogen, and decreased blood urea nitrogen (BUN) and malondialdehyde (MDA) content compared to control groups ${ }^{40}$. In addition, according to a recently published study, when SC ingredients were ingested over a long time in chronic fatigue mice, learning and memory abilities partly improved through the regulation of the Nrf2/Keap1/ ARE signaling pathway. This study confirmed the interaction of 30 related active compounds and 21 genes through network analysis. Therefore, we suggest that the most prominent benefits of SC intake are improved metabolism, enhanced cognitive abilities, and reduced chronic fatigue.

In conclusion, based on the current results, we suggest that the consumption of SC may have beneficial effects on health. The active compounds of SC may be considered as potential pharmacological candidates through the activation of diverse pathways such as postsynaptic neurotransmitter receptor activity, an intracellular steroid hormone receptor signaling pathway, and endopeptidase activity owing to their synergistic benefits with exercise.

\section{ACKNOWLEDGEMENTS}

This work was supported by a National Research Foundation of Korea (NRF) grant funded by the Korean government (MSIT) (NRF-2020R1F1A1049665). This study was supported by the KU Research Professor Program of Konkuk University.

\section{REFERENCES}

1. Jaul E, Barron J. Age-related diseases and clinical and public health implications for the 85 years old and over population. Front 
Public Health. 2017:5:335.

2. Majmundar MK, Hayward MD, editors. Washington (DC): National Academies Press (US). Future directions for the demography of aging: proceedings of a workshop. National Academies of Sciences, Engineering, and Medicine; Division of Behavioral and Social Sciences and Education; Committee on Population. 2018.

3. Meng Q, Lin MS, Tzeng IS. Relationship between exercise and alzheimer's disease: a narrative literature review. Front Neurosci. 2020;14:131.

4. Andersen K, Rasmussen F, Held C, Neovius M, Tynelius P, Sundstrom $\mathrm{J}$. Exercise capacity and muscle strength and risk of vascular disease and arrhythmia in 1.1 million young Swedish men: cohort study. BMJ. 2015;351:4543.

5. Song $\mathrm{C}$, Ikei $\mathrm{H}$, Miyazaki Y. Physiological effects of nature therapy: a review of the research in Japan. Int J Environ Res Public Health. 2016;13:781.

6. Bach HV, Kim J, Myung SK, Cho YA. Efficacy of ginseng supplements on fatigue and physical performance: a meta-analysis. $J$ Korean Med Sci. 2016;31:1879-86.

7. Kimura I. Medical benefits of using natural compounds and their derivatives having multiple pharmacological actions. Yakugaku Zasshi. 2006;126:133-43.

8. Oikawa SY, Brisbois TD, van Loon LJC, Rollo I. Eat like an athlete: insights of sports nutrition science to support active aging in healthy older adults. Geroscience. 2021:43:2485-95.

9. Tvrdá E, Michalko J, Árvay J, Vukovic NL, Ivanišová E, Duuračka M, Matušíková I, Kačániová M. Characterization of the omija (Schisandra chinensis) extract and its effects on the bovine sperm vitality and oxidative profile during in vitro storage. Evid Based Complement Alternat Med. 2020;2020:7123780

10. Han HJ, Jung UJ, Kim HJ, Cho SJ, Kim AH, Han Y, Choi MS Combined supplementation with grape pomace and omija fruit ethanol extracts dose-dependently improves body composition, plasma lipid profiles, inflammatory status, and antioxidant capacity in overweight and obese subjects. J Med Food. 2016;19:170-80.

11. Lee JH, Lee YY, Lee J, Jang YJ, Jang HW. Chemical composition, antioxidant, and anti-inflammatory activity of essential oil from omija (Schisandra chinensis (Turcz.) Baill.) produced by supercritical fluid extraction using CO2. Foods. 2021;10:1619.

12. Poudel S, Lee Y. Impaired taste associative memory and memory enhancement by feeding omija in parkinson's disease fly model. Mol Cells. 2018,41:646-52.

13. Ru J, Li P, Wang J, Zhou W, Li B, Huang C, Li P, Guo Z, Tao W, Yang Y, Xu X, Li Y, Wang Y, Yang L. TCMSP: a database of systems pharmacology for drug discovery from herbal medicines. $J$ Cheminform. 2014:6:13.

14. Cho S, Hong R, Yim P, Yeom M, Lee B, Yang WM, Hong J, Lee HS, Hahm DH. An herbal formula consisting of Schisandra chinensis (Turcz.) Baill, lycium Chinense mill and eucommia ulmoides oliv alleviates disuse muscle atrophy in rats. J Ethnopharmacol. 2018;213:328-39.

15. Jung S, Moon HI, Kim S, Quynh NTN, Yu J, Sandag Z, Le DT, Lee $\mathrm{H}$, Lee $\mathrm{H}$, Lee MS. Anticancer activity of gomisin J from Schisandra chinensis fruit. Oncol Rep. 2019;41:711-7.

16. Shao S, Wang MX, Zhang HY, Fan L, Han RX, Shen YX, Yan $M M$, Zhao DQ. Antifatigue activity of glycoprotein from Schisandra chinensis functions by reducing oxidative stress. Evid Based Complement Alternat Med. 2020;4231340.
17. Park J, Han S, Park H. Effect of Schisandra chinensis extract supplementation on quadriceps muscle strength and fatigue in adult women: a randomized, double-blind, placebo-controlled trial. Int $J$ Environ Res Public Health. 2020;17:2475.

18. Hartgens $\mathrm{F}$, Kuipers $\mathrm{H}$. Effects of androgenic-anabolic steroids in athletes. Sports Med. 2004;34:513-54.

19. Louis J, Hausswirth C, Bieuzen F, Brisswalter J. Muscle strength and metabolism in master athletes. Int J Sports Med. 2009;30:754-9.

20. Hargreaves M, Spriet LL. Skeletal muscle energy metabolism during exercise. Nat Metab. 2020;2:817-28.

21. Yeon M, Choi $H$, Jun HS. Preventive effects of Schisandrin $A$, a bioactive component of Schisandra chinensis, on dexamethasone-induced muscle atrophy. Nutrients. 2020;12:1255.

22. Scheffer DDL, Latini A. Exercise-induced immune system response: anti-inflammatory status on peripheral and central organs. Biochim Biophys Acta Mol Basis Dis. 2020;1866:165823.

23. Cerqueira E, Marinho DA, Neiva HP, Lourenco O. Inflammatory effects of high and moderate intensity exercise-a systematic review. Front Physiol. 2019;10:1550.

24. Furman D, Campisi J, Verdin E, Carrera-Bastos P, Targ S, Franceschi C, Ferrucci L, Gilroy DW, Fasano A, Miller GW, Miller AH, Mantovani A, Weyand CM, Barzilai N, Goronzy JJ, Rando TA, Effros RB, Lucia A, Kleinstreuer N, Slavich GM. Chronic inflammation in the etiology of disease across the life span. Nat Med. 2019;25:1822-32

25. Luo G, Cheng BC, Zhao H, Fu XQ, Xie R, Zhang SF, Pan SY, Zhang Y. Schisandra chinensis lignans suppresses the production of inflammatory mediators regulated by NF-KB, AP-1, and IRF3 in lipopolysaccharide-stimulated RAW264.7 Cells. Molecules. 2018;23:3319.

26. Ertek S, Cicero A. Impact of physical activity on inflammation: effects on cardiovascular disease risk and other inflammatory conditions. Arch Med Sci. 2012;8:794-804.

27. Hurt RT, Kulisek C, Buchanan LA, McClave SA. The obesity epidemic: challenges, health initiatives, and implications for gastroenterologists. Gastroenterol Hepatol (NY). 2010;6:780-92.

28. Barnes AS. The epidemic of obesity and diabetes: trends and treatments. Tex Heart Inst J. 2011;38:142-4.

29. Paschos P, Paletas K. Nonalcoholic fatty liver disease and metabolic syndrome. Hippokratia. 2009,13:9-19.

30. Tinajero MG, Malik VS. An update on the epidemiology of type 2 diabetes: a global perspective. Endocrinol Metab Clin North Am. 2021;50:337-55

31. Leal LG, Lopes MA, Batista ML, Jr. Physical exercise-induced myokines and muscle-adipose tissue crosstalk: a review of current knowledge and the implications for health and metabolic diseases. Front Physiol. 2018:9:1307.

32. Seo DY, Park SH, Marquez J, Kwak HB, Kim TN, Bae JH, Koh JH, Han J. Hepatokines as a molecular transducer of exercise. J Clin Med. 2021:10:385

33. Carr RD, Solomon A. Inhibitors of dipeptidyl peptidase-4 as therapeutic agents for individuals with type 2 diabetes: a 25-year journey. Diabet Med. 2020;37:1230-3.

34. Itou M, Kawaguchi T, Taniguchi E, Oriishi T, Sata M. Dipeptidyl peptidase IV inhibitor improves insulin resistance and steatosis in a refractory nonalcoholic fatty liver disease patient: a case report. Case Rep Gastroenterol. 2012;6:538-44.

35. Li YP, Xiao J, Liang X, Pei Y, Han XF, Li CX, Tian H. DPP. 
4 inhibition resembles exercise in preventing type 2 diabetes development by inhibiting hepatic protein kinase Cepsilon expression in a mouse model of hyperinsulinemia. $J$ Int Med Res. 2020;48:300060520934635.

36. Carroll TJ, Taylor JL, Gandevia SC. Recovery of central and peripheral neuromuscular fatigue after exercise. J Appl Physiol (1985). 2017;122:1068-76.

37. Meeusen R, Roelands B. Central fatigue and neurotransmitters, can thermoregulation be manipulated? Scand J Med Sci Sports. 2010;20:19-28.

38. Friend DM, Devarakonda K, O'Neal TJ, Skirzewski M, Papazoglou I, Kaplan AR, Liow JS, Guo J, Rane SG, Rubinstein M, Alvarez VA, Hall KD, Kravitz A. Basal ganglia dysfunction contributes to physical inactivity in obesity. Cell Metab. 2017;25:312-21.

39. Szopa A, Ekiert R, Ekiert H. Current knowledge of Schisandra chinensis (Turcz.) Baill. (Chinese magnolia vine) as a medicinal plant species: a review on the bioactive components, pharmacological properties, analytical and biotechnological studies. Phytochem Rev. 2017;16:195-218.

40. Shao S, Wang MX, Zhang HY, Fan L, Han RX, Shen YX, Yan MM, Zhao DQ. Antifatigue activity of glycoprotein from Schisandra chinensis functions by reducing oxidative stress. Evid Based Complement Alternat Med. 2020;2020:4231340.

41. Kim KY, Ku SK, Lee KW, Song CH, An WG. Muscle-protective effects of Schisandrae fructus extracts in old mice after chronic forced exercise. J Ethnopharmacol. 2018;212:175-87.

42. Cho YH, Lee SY, Lee CH, Park JH, So YS. Effect of Schisandra chinensis Baillon extracts and regular low-intensity exercise on muscle strength and mass in older adults: a randomized, double-blind, placebo-controlled trial. Am J Clin Nutr. 2021;113:1440-6. 